\title{
Application of Agile Methods on Embedded System Development Jun Xiao ${ }^{1, a}$ \\ ${ }^{1}$ Xi'an Aeronautical University, Xi'an, Shaanxi, 710077 \\ ${ }^{\mathrm{a}}$ email
}

Keywords: Embedded Systems Development, Agile Methods, Applied Research

\begin{abstract}
At present, the rapid development of computer technology, embedded system software has been widely used, with the increase in application volume, which faces a number of system development cost, quality and other aspects of pressure. In order to increase productivity, improve the ability to adapt to changing requirements, Agile methods gradually be applied to traditional software projects. In this paper, the embedded system development projects analog implementation interphone serial debug information module as an example, the application of agile methods and practical application.
\end{abstract}

\section{Introduction}

Agile development, it can be adapted to the needs of users, their needs change as the core, iterative, etc. to carry out an incremental development approach. In agile methods, software development project is divided into a plurality of sub-projects, the outcome of multiple subprojects can be tested, although a single subproject but can run independently, and ultimately the formation of integrated project works best [1]. This development approach conducive to the development of improved efficiency, attention to their response to changes and achieve customer value. In this paper, the method carried out relevant research.

\section{The Concept of Embedded System Development and Application Overview}

Embedded systems, it applies to the strict requirements of the dedicated computer system, the so-called strict requirements mainly refers to the cost, reliability, power consumption and size, etc. required. Computer systems technology is its underlying technology, with the objective. Hardware and software can be cut, its constituent elements there are four main aspects, one embedded microprocessor, the second is the embedded operating system, the third is the user's application, the four peripheral hardware equipment, etc. [2]. Application of the system can be monitored on other equipment, control and management. Specific based hierarchy shown in Fig.

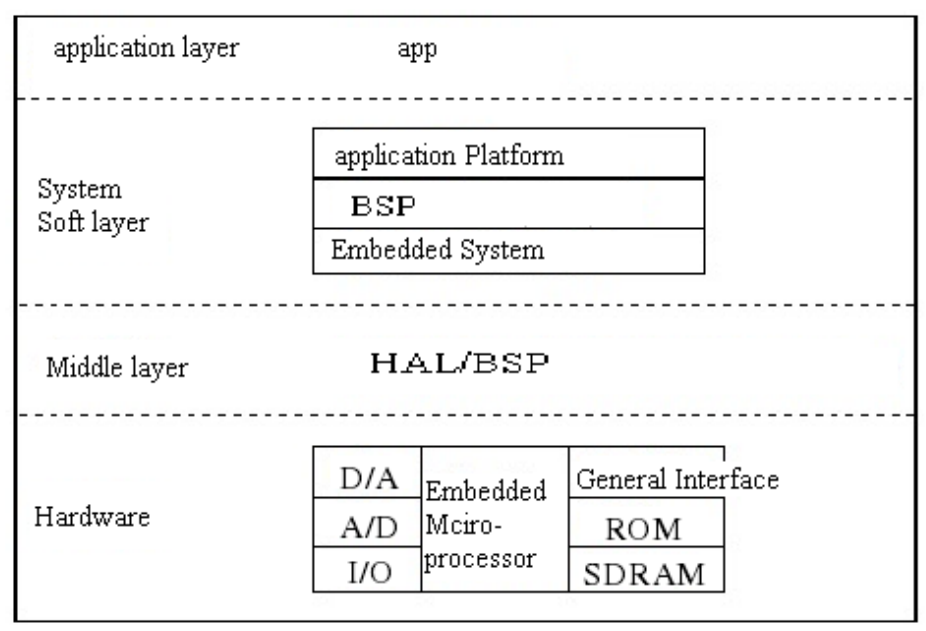

Fig.1 Embedded system level architecture

Embedded systems, it is a special-purpose computer system designed for specific applications and multi-applications, whether in the actual design of the system development will be subject to 
many restrictions. More restrictions from existing software, hardware platform restrictions, there are some time constraints, that is, to meet real-time requirements. To compensate for these limitations, the system under development will consume a lot of costs and resources. In recent years, with the improvement of technology and associated computer hardware equipment performance, this system has been widely used applications gradually expanded, and the scale of its own software is also gradually increasing software content more complicated. Long past the difficulty caused by the embedded system in the actual development greatly enhanced. At present, more and more people began to focus on the quality of its system, the system of cost control and project cycle began to take it seriously. On the current development, how to develop a high quality of embedded systems has become one of the important issues of development software development industry.

\section{An Overview and the Development Status of the Concept of Agile Methods}

Agile methods, is a new approach to software development, its biggest advantage is that it can quickly respond to the changing needs of software development, cooperation and the exchange of ideas between engineers and programmers and other related personnel and team [3]. Such exchanges close and frequent changes in demand implementation code written in collaboration by the team, the whole process has a certain method of team organization, attention to personal fulfillment.

Agile methods, after years of practice tests, to this day has been basically achieved great success in many software development projects are to be applied. And have achieved good application results. After practice proved agile methods effectively reduce development costs, enhance productivity, and shorten the development cycle. Agile methods can effectively compensate for embedded system development difficulties, the team can be a very good ability to adapt to changing requirements. Together with its advantages of the method itself, you can always deal with all kinds of challenges faced by embedded systems.

\section{Agile Development Methods in Embedded System Development}

Analog radio project is embedded system project, debug information module in which the role of the serial port for standard analog radio system to provide information output. This module because its function is more independent and require urgent to carry out the project, the more practical application of agile methods. At the same time, information about the module output is to run information systems, this output facilitates software developers and other systematic tracking and analysis.

Creative final to determine a good user of the module after the agile development method must first collect user information and material, and then a list of material gathered together for Creative Leadership or filter selected portions of some of the test personnel department, screening [4 ]. This is the first mission requirements gathering, followed by the final screening out material decomposition. The relatively large number of material and information broken down into multiple smaller clips, little information, but too small for those materials can be integrated into a larger creative. After the establishment of iterations. Iterative period is one week, to establish four iterations of the total amount of material and material content. Finally, the integration and development of the creative task decomposition time table and operating procedures, which also includes tracking the progress of content.

Traditional project developers, engineers or more testers will first write the code, then use the code coverage test cases, so it is prone to problems, the problem of missing phenomenon on coverage of test cases, investigate its causes mostly because the test code and developers who code is caused by inconsistent [5]. The agile method is applied to test-driven development can effectively avoid or reduce such problems. Agile development methods, the main application is the first process in the programming designed to test the program before, missing only when a line of code in the program code that can be added. The advantage of this approach is that you can test each function, you can pass the test to verify the correctness of the operation or not. In addition, pre-prepared test 
cases allows engineers to use different observation points, and the program is designed to measure, releasing software coupling. Because the test is a form of documentation, enforceable, it can be compiled, so more reliable.

Engineers conducting conventional procedures and systems according to user needs directly to the development of coding operation, while writing code edges prepared to consider, but it is completely different agile methods, agile methods advocated to write test cases. After practice shows that many projects are represented to write test cases for too long, sometimes often exceed the time code is written so that some engineers feel a waste of time, but actually not the case, writing test cases written in a long time in the short term, but with the number of skilled and writing skills to write, time will be faster, and the use of written software development quality after cases significantly higher than traditional direct coding quality. Meanwhile, the test can be compiled and run, so adding new code, test cases using the original interface, a new code of the original impact test is possible, thus effectively faster debug, with the the code is gradually increased, the more obvious advantages. And the test case is a document, that document can exist independently, and the code can be updated to keep pace.

Traditional software development process is no reconstruction of this part. Past practice after the completion of the preparation of the code can be verified. Verification process will always be progress in order to meet the requirements of the direct delivery of the phenomenon. This phenomenon can cause long past complicated code with the code growing, eventually leading to a logical system errors, resulting in the use of personnel do not understand the procedures and systems. The agile method is applied to the development of the module, which is an important part of the reconstruction. Reconstruction after the code refers to the part of the completion of the preparation. Agile methods require after the code needs to be run to complete the test, after confirmation test can be reconstructed. Reconstruction is on the development of the system of duplicate code, complex code, the code is unclear and fuzzy logic code Shanfanjiujian. Ensure the correct application of the code based on the simplified code, this simplified process is called remodeling. Reconstruction and commented in fact, the code can help system developers design optimization. This reconstruction method can boot code moving in a positive direction, it takes significantly shorter than the time-consuming traditional methods of correcting complicated code.

Iteration, in fact, is a kind of cycle, after each iteration can be achieved on the existing systems and procedures for the operation and demonstration. After a time of iteration ends, customers can be run on the current drill program to watch. Because the existing operational procedures, operations, so customers can make the appropriate comments and suggestions for improvement during the period of the existing program. Its program performance and program functions can make timely adjustments. But it can also increase the corresponding new material. Iterative process may further adjust the system development pace to achieve the adjustment of the system to predict the development plan and amendments, system developers can be made according to customer needs and to develop estimates. The modules were set up four iterations, these four iterative process to achieve the expected within functions and successfully be applied. In this study, the developer after the completion of each iteration are for customers exercise program to meet customer expectations design objectives, of which the customer also made suggestions to improve timely adjusted according to customer demand and engineers amendment ultimately meet the overall needs of customers.

\section{Conclusion}

In the actual programming and software development engineers and more will make some design, coding and other aspects of the errors, once these problems, the traditional waterfall model and other methods appear more difficult to continue, but after applying agile methods can be an iterative manner fast problem identification and problem correction. Since all the code development project engineers working together to complete, so the enhanced teamwork skills, enhanced coordination and communication between the teams, greatly improving the work efficiency of the software development team, and its value significantly. 


\section{References}

[1] Rong Guoping, Liu Tianyu, Xieming Juan,;A Summary of Research embedded systems development in agile methods [J]. Journal of Software 2014, 25 (2): 267-283.

[2] Lu Jinhua, Wei Hui. Agile development in embedded programming Application and Practice [J]. electronic world, 2014 (13): 84-84.

[3] Xie Xiaoyun embedded software development based on agile greenhouse gateway and multi-V model [J]. Electronics and Software Engineering, 2015 (1): 75-76.

[4] Zhang Yue, Jin Hui. Agile Development Methodology [J]. embedded in the Teaching Reform of Nanjing Radio and Television University, 2012 (2): 43-48.

[5] Liu Xi. Exploratory software testing method and its application in embedded systems [J]. Modern electronic technology, 2014 (20): 74-79. 